\title{
Decision Traps and Improvement of Decision Power
}

\author{
Xiangyu Chen \\ School of Public Affairs and Administration \\ University of Electronic Science and Technology of China \\ Chengdu, China
}

\begin{abstract}
After the outbreak of crisis, both enterprises and governments require decision-makers to make quick decisions to effectively control the scale of the crisis. In the face of an imminent peril, the behavioral habits and psychological factors of decision makers are very important. Any carelessness may lead to cognitive and action errors and then decision traps. Since these decision-making habits and behaviors are highly elusive, in order to avoid decision-makers from falling into the wrong judgment, that is, the decision trap, while thinking they are making a good choice, it is necessary to make a correct decision, act quickly and make timely improvement to the decision-making scheme from the collective to the decisionmaker. Otherwise, underlying dangers will easily cause the failure of the project.
\end{abstract}

Keywords—decision traps; group decision making; cognitive trap; stereotypical thinking

\section{INTRODUCTION}

In the age of information explosion, it is impossible for people to accept all information completely. They usually choose based on their own experience and preference, and instinctively exclude suggestions and opinions that are contrary to their own ideas, even if there is some useful information. At the same time, they will also choose the examples that can support their decision opinions to convince themselves of the correctness of their decision, which is called selective cognition in psychology. Selective cognition is not all bad. When decision-makers and their groups are in a complex and information-flooding environment, they often need to rely on selective cognition to help them quickly understand the background and key information of the problem to be solved so as to make effective decisions in a timely manner. However, this kind of decision-making dominated by selective cognition is influenced by the decision maker's stereotypical thinking, and inevitably falls into decision traps due to the surrounding environment, personal preference and other factors. Taking the company's project planning as an example, due to the information resources of the advantages and disadvantages of the project and party B's eagerness to sign the contract, the feasibility and profit rate of the project are often artificially inflated, which is not consistent with the actual situation. However, when such background information is "brainwashing" around the decision-maker, he or she can easily show excessive attention and conviction to the above project information, thus forming psychological dependence and falling into the decision trap. Especially in a group, if the teams composed of experienced managers all rely on selective cognition or conformity, they will also make naive mistakes in making important decisions, leading to the failure of decisions, which can be refined as a collective decision trap.

\section{FACTORS CONTRIBUTING TO DECISION TRAPS}

Stereotypical thinking is a way of thinking that people often use to solve problems in daily life. On the one hand, the past successful experience can be used to draw lessons; on the other hand, decision-making time and cost can be shortened, decision-making risks can be more or less reduced, and the feasibility and success rate of decision-making can be enhanced. ${ }^{1}$ There is no denying that the conventional way of thinking can provide decision-makers with meaningful information and evidence. Especially for logistics and transportation projects, construction projects, mechanical construction projects and other repetitive and analogical work, the positive role of stereotype thinking in timely decision-making cannot be ignored. Due to the differences in the background, relevant environment, operation mode and other internal factors of each project, its uniqueness determines that no two projects are exactly the same, even though they appear to be identical on the surface, there are essential differences in essence.

Moreover, as controversial as group decision-making is, it comes from groupthink, which refers to the highly consistent thinking patterns of the members of a group. When making decisions, group members put the unity and cohesion of the team above any other reference factors, which often leads to disregard for other reasonable or even better alternatives and the overall efficiency of group thinking and the accuracy of judgment, are reduced due to external pressure. ${ }^{2}$ Although it has been pointed out that the decision-making mode represented by collective cohesion leads to excessive pursuit of behavioral consistency, the external manifestations of collective thinking are various. First, the over-reliance on the collective will subconsciously produce the illusion that the decision is perfect and idealize the decision-making behavior excessively. Second, the decision maker may believe or have prejudice to the whole group of personnel, that is, to form a closed fixed thinking.

\footnotetext{
Yang Chuanjun, Yu Xiaozhong, Psychological traps in project decision-making process. Decision Reference. 2004. (5).

Ke Shenghua, Skipping the Decision Trap. Finance and Accounting Monthly. 2010 (1)
} 
Third, under the external pressure of conformity, collective members will form consistent ideological guard or introverted pressure based on crowd mentality, which will cause great psychological pressure to decision makers. This huge pressure of conformity usually makes the decisionmaker rush to make a decision or wait for others to make a decision to make their own position, which reduces the decision-making efficiency to a certain extent.

Besides internal reasons, decision-making environment is also an important factor for the rise of decision traps. The decision-making environment includes but is not limited to economy, politics, ecology, population, etc. It can also be called the background environment accompanied by decision-making problems and is the sum of direct or indirect factors acting on decision-making behaviors. Due to its certain randomness, fuzziness and unpredictability, emergency decision-making reacts on the external environment and has great uncertainty, which is manifested in behavior state, subjective cognition and consequence influence, and exists outside the collective boundary and exerts varying influences on the collective external environment. Corresponding to this is the internal environment, which is the constituent elements of the collective, such as the collective members, material conditions and all kinds of soft power. With the development and change of external conditions, the internal environment of decision-making changes at every moment. Once the vicious development of the internal environment begins, it will have internal negative impact on decision makers and bring them into the decision trap. Psychological environment mainly refers to the mental quality of decision-makers in the face of external pressure such as public opinion, which is greatly influenced by external environment.

\section{THE REFLECTION AND INFLUENCE OF DECISION TRAPS IN LIFE}

The causes of decision traps have been analyzed above, and they are also very common in life, which may lead to decision failure if not paid attention to. One of the decision traps is the "Hobson choice trap", which is characterized by the inability to skillfully face the sudden crisis situation and deal with the messy and complicated information under huge psychological pressure. With the thinking mode getting immobilized and unidirectional, the decision makers are unable to see the real situation of the objective environment driven by the inertia of choice. Because of the contradiction between the perspective of thinking choice and the objective standard, the behavior of decision makers tends to be closed, which is difficult for them to stimulate their own creativity and subjective initiative, and thus hard to find the right perspective and method to solve the problem. For example, when faced with two piles of straw, a donkey always walks to one side thinking that the straw in the other pile of is more and better, thus running from both sides repeatedly, and finally being starved to death. This seemingly absurd choice with no alternative is a typical decision trap of "Hobson choice". There is a similar example in ancient China. In "Zhuangzi · On the Equality of Things", a story is described as follows. There was a monkey keeper who received strong dissatisfaction when he suggested feeding the monkeys three acorns in the morning and four in the evening. But then he made the monkeys satisfied by feeding them four in the morning and three in the evening. This is where the phrase "morning three night four" comes. The total number of acorns didn't change, but the monkeys thought they could eat more food in the second form. This is a reflection of Hobson's choice mindset.

Another typical trap is the "group decision making" trap, also known as "collective blindness". Even if a team is made up of members with high IQ and ability, in a particular situation, especially in a crisis situation when internal pressure catalyzing the group increases, the crowd mentality makes the mental level, ability to analyze objectively, and ability to judge right from wrong of the policy makers team greatly reduced. At this time, there is a tendency among the members to reduce or filter out the "misfit" views so that the final decision is consistent, especially with the top decision makers. ${ }^{3}$ The original purpose of "group decision making" is to pool the wisdom of the masses and gather individual strength to improve the feasibility of decision-making. However, the false consensus will turn "group decision making" into a decision trap, and the stronger the collective cohesion is, the easier it will be to form Herd Effect, that is, to blindly make group decisions without considering other reasonable or even better alternative offer. This is closely related to China's traditional culture, where Chinese people value harmony and over-emphasize "harmony", while the idea of individual uniqueness is drowned out. Even if these ideas are reasonable and feasible, people may not dare to express their disagreement with their superiors as they tend to follow their superior leaders blindly. The curse of "collective blindness" puts dissidents under pressure from other members of the group and they have to conform to group decisions only by remaining silent or changing their views 4 to avoid "discordance". In essence this is not conducive to improving the scientificity and feasibility of decision-making. For example, Kennedy's invasion of Cuba in the "Pegasus Bay Incident", Vietnam War in the 1960s and the US "Challenger" space shuttle accident in 1986 and other events, were absurd choices made by elite teams caught in the trap of "group decision-making".

In addition to the two typical traps mentioned above, the comparison trap is also very common in daily life. People are naturally inclined to compare, and the resulting stereotype is one of the common decision-making biases in society. Taking consumers' views on bundled products and willingness to pay as an example, under the effect of stereotypes, the riding effect of brands is very obvious. ${ }^{5}$ Consumers tend to increase their consumption willingness to bundled or combined products because the main product has

Dong Jianmeng, Zhu Ruibo. Common Traps and Improvement of Decision-making Power in Public Crisis Decision-making, Leadership Science. 2013(5).

4 Shi Xiaoyu, Gu Qinxuan. "Team Thinking - the Mystery of Giving Full Play to Team Wisdom". Journal of Intelligence. 2015 (9).

Zhang Quancheng, Lu Dong, Zhou Tingrui. A Review of Literature on Context Effect in Custom Choice Behavior and Future Research Prospect. Soft Science. 2011, (10). 
the characteristics of well-known brand, high quality and understandability, and pay for the whole bundled product, even if the subsidiary product does not have good quality or value. ${ }^{6}$ This is because when people are faced with a choice to think, the brain tends to make trade-offs or comparisons, but is not good at directly measuring or evaluating things. Therefore, people tend to take a certain one or a certain kind of things as a reference when making decisions, and then make comparisons and finally make choices or judgments. People like not only to compare things, but also to compare themselves and others. The general cognition of people is that without comparison or contrast, it is difficult for them to make value judgment and decision independently. However, on the one hand, the application of habitual comparative thinking to life usually leads to numerous cognitive biases in decision-making judgment of behavioral individuals that cannot be eliminated. On the other hand, due to the different choice of reference, the final decision is inconsistent with the ideal result expected in advance.

\section{WAYS TO AVOID DECISION TRAPS}

To improve the decision-making level, it is necessary to establish a correct and scientific decision-making view, optimize the information support system, improve the operation mode of team decision-making, reduce formalism and bureaucracy, and enrich the methods and tools of decision-making.

The decision-making body and the system are organically combined to form the decision-making mechanism, in which the individuals or groups participating in the decisionmaking link and performing the decision-making responsibilities are the decision-making body, and a series of systems guiding and standardizing the decision-making behavior are the decision-making system. From the perspective of subjects, there are central decision system, consultation support system, decision information system and decision monitoring system, as well as the decision environment system considering the extension. The five systems are organically combined to form a decision-making community, which evaluates and monitors the schemes and finally facilitates the formation of decisions. ${ }^{7}$ The main function of the decision-making mechanism is to regulate the procedure and method of decision-making and the authority of the subject's behavior. Abuse of rights is restrained by external norms. The more rigorous a decision-making system is, the less likely the subject is to make mistakes.

Although the existence of group is an objective fact, group thinking is not harmful without benefit. Through giving full play to subjective initiative, the disadvantages of group thinking can be overcome and the trap of "group decision-making" can be avoided. The successful experience of many teams from ancient times to now provides the best proof of overcoming the crowd mentality. In fact, the reason why a group with high intelligence will fall into "decision

\footnotetext{
Zhong Jichuan. Trade-off Trap Under Stereotype Bias: Experiment on Bundling Strategy, 2018 (1).

Zhang Chuanzhou. "Groupthink Trap" Governance. Management Forum. 2016(1).
}

traps" is largely due to the excessive emphasis on the influence of "accordance" in the group and the neglect of the unique perspectives and innovative ideas of individuals. Therefore, in order to avoid the above phenomenon, it is necessary to start from the bottom of the thinking model, that is, to guide the thinking mode of individuals in a group through the establishment of beliefs and self-dialogue. ${ }^{8}$ To be specific, what needs to be done includes, to establish a collective common belief system, pursue "harmony in diversity" rather than "unharmonious accordance", motivate the team to have internal dialogue, allow each member to have the opportunity to display their talents, and reward the members to continue to challenge the current predicament. ${ }^{9}$ Motivating teams to engage in internal dialogue is an effective tool to improve decision-making performance. In groups where groupthink prevails, effective communication mechanisms can be undermined and members who are keen to express different views will come under pressure from other members of the group. In order to maintain the unity and consistency pursued by the group, members with different views can only abandon their own opinions even if they are correct. ${ }^{10}$ So, it is necessary to respect different opinions, and by drawing a blueprint for the future to combat external decision-making pressure, to break down the wall of thinking.

\section{CONCLUSION}

People need to make all kinds of decisions in life, so how to make good decisions and how to avoid falling into decision traps are very important. This paper analyzes the factors of decision trap, the manifestation and influence of decision traps in life, and finally puts forward scientific methods to avoid it, in hope that people can improve their decision-making level and manage their lives well.

\section{REFERENCES}

[1] Yang Chuanjun, Yu Xiaozhong. Psychological Traps in Project Decision-making Process. Decision Reference. 2004. (5). (in Chinese)

[2] Ke Shenghua. Skipping the Decision Trap. Finance and Accounting Monthly. 2010 (1). (in Chinese)

[3] Dong Jianmeng, Zhu Ruibo. Common Traps and Improvement of Decision-making Power in Public Crisis Decision-making. Leadership Science. 2013(5). (in Chinese)

[4] Shi Xiaoyu, Gu Qinxuan. "Team Thinking — the Mystery of Giving Full Play to Team Wisdom". Journal of Intelligence. 2015(9). (in Chinese)

[5] Zhang Quancheng, Lu Dong, Zhou Tingrui. A Review of Literature on Context Effect in Custom Choice Behavior and Future Research Prospect. Soft Science. 2011, (10). (in Chinese)

[6] Zhong Jichuan. Trade-off Trap Under Stereotype Bias: Experiment on Bundling Strategy, 2018 (1). (in Chinese)

[7] Zhang Chuanzhou. "Groupthink Trap" Governance. Management Forum. 2016(1). (in Chinese)

\footnotetext{
8 Wang Yuan. The art of moderation in leadership decisionmaking [J]. Leadership Science, 2017 (22): 17-18.

Dong Jie. Strategies for the use of "information power" in leadership decision-making. Art of Leadership, 2019 (1).

10 Zhu Liyan, Sun Jian. Art and Science of Leadership (2nd edition) [M]. Wuhan: Huazhong University of Science and Technology Press, 2013.
} 
[8] Wang Yuan. The art of moderation in leadership decision-making [J]. Leadership Science, 2017 (22): 17-18. (in Chinese)

[9] Dong Jie. Strategies for the use of "information power" in leadership decision-making. Art of Leadership, 2019 (1). (in Chinese)

[10] Zhu Liyan, Sun Jian. Art and Science of Leadership (2nd edition) [M]. Wuhan: Huazhong University of Science and Technology Press, 2013. 